\title{
A Survey - Energy Efficient Routing Protocols in MANET
}

\author{
Jyoti Upadhyaya and Nitin Manjhi \\ Department of Computer Science, RGPV University \\ Shriram Group of Colleges, Banmore, Madhya Pradesh, India \\ jyotiupadhyaya23@gmail.com,nitin.manjhi@gmail.com
}

\begin{abstract}
The field of mobile ad hoc network (MANETs) has gained an important of the researchers and become very popular in last year's. Mobile Ad hoc networks is a network that can change locations and configure itself; as it is infrastructure less and have no central administration. MANET nodes are mobile and battery powered and has limited energy resources. Life of nodes is directly proportional to the battery in the device operating at the node. Minimize energy consumption and maximize life of network is still an immerging issue of MANET. In order to enhance the lifetime of the Ad hoc network; various energy efficiency techniques are needed. This paper presents a survey on energy efficient routing protocols. These algorithms and methodologies modify routing protocol in order to reduce energy consumption and maximize life of network. These proposed methodologies also minimize energy consumption by selecting an energy efficient route.
\end{abstract}

\section{Introduction}

An ad-hoc network is a collection of wireless mobile nodes set up a network without the need of any centralized administration or fixed network infrastructure. Mobile ad-hoc networks are self-organizing and self-configuring multihop wireless networks [1], in which nodes collaborate with each other by forwarding packets for communication. Due to mobility of nodes, network has dynamic topologies. As this network have no centralized administration, the nodes in the network can both act as a router or hosts to send packets in the networks. One node can communicate with the other node that may not lie within in the transmission range of one another. Routing is one of the major problems of networking to deliver packets from one node to the other in the network. A Manet's uses peer-to-peer multihop routing to provide network connectivity. MANETs can be characterized as having dynamic, multihop, and rapid changing topology [2]. There are lots of issues in designing an ad-hoc network. Because of rapid changing topology, nodes changes its position on every second this is one of the major challenge is link failure. Route selection can also be considered as one of the major issue in routing protocol. Selecting non-optimal routes may increase delays, routing loads, decrease throughput and increase loss rate since the selected route will break quickly or nodes get congested. Most of the traditional ad hoc routing protocols use hop count as a metric for selecting paths from source to the destination. However, the shortest path may not always be the optimal way for selecting paths. Since shortest paths may contain congested nodes or nodes having weak connectivity degree among each other. Hence other factors must be taken into consideration while selecting routes like the link quality and the nodes situation at a certain time. Communication, between non-neighboring nodes, requires a routing protocol, so a stable and efficient routing method is required for longer live transmission. 


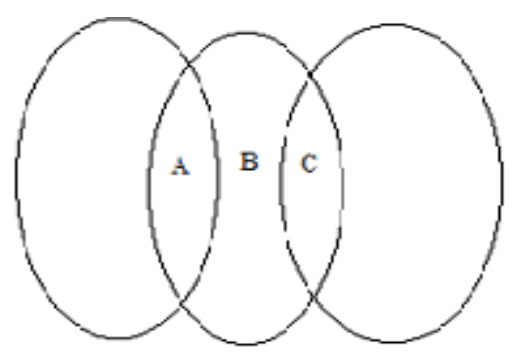

Figure 1. Example of a Simple Ad-Hoc Network

A. Problems with Routing in MANETs

1) Asymmetric Links: In ad-hoc networks, as the nodes are mobile and keep changing their location in the network, so it does not rely on symmetric links as in the case of wired network.

2) Dynamic Topology: Nodes in the network are free to move; hence network topology may change unpredictably and randomly over time.

3) Routing Overhead: In ad-hoc network nodes may change their position in the network. So, there is a possibility that some stale routes are generated in the routing table which leads to unnecessary routing overhead.

4) Inference: As links come and do depending on the transmission characteristics, one transmission might get collide with other and node may overhear the transmission of other and can corrupt the whole transmission.

5) Limited Power Supply and Radio Range: Wireless links have lower capacity than wired networks. Due to limited power supply MANETs have limited radio range.

6) Energy Consumption: Nodes in the mobile ad-hoc networks heavily depends on battery life for their energy. To maximize the lifetime of network energy conservation is the key challenge of MANET.

7) Mobility-induced Route Changes: As nodes in the ad-hoc networks are mobile; hence on-going session suffers link failure. This often leads to route changes.

8) Security: MANET is prone to eavesdropping and security attacks than infrastructure networks because of its infrastructure less nature.

B. Energy Efficiency Issues

Ad-hoc networks are energy constrained since nodes reply on battery power. Due to limited power supply if some nodes die early they cannot communicate further more. Hence, energy conservation is still a key challenge in MANET in order to enhance the lifetime of network and maximize the use of energy. Following are the issues why energy efficient routing is needed in mobile ad-hoc networks:

- Limited energy reserve.

- Difficulty in replacing batteries.

- Lack of central coordination.

- Constraints on the battery resources.

- Selection of optimal transmission power.

C. Advantages of MANET

The advantages of mobile ad-hoc networks are as follows:

- Independent because of having no central coordination. Nodes can act both as routers or hosts.

- Self-configuring network; can setup wherever needed. 
- Access information regardless of geographical environment.

- Flexible to highly dynamic topologies.

- Robust

- Less expensive than wired networks.

D. Applications of MANET

1) Military Applications: Ad-hoc wireless networks are useful in establishing communication in a battle field.

2) Collaborative and Distributed Computing: A group of people in a conference can share data in ad-hoc networks.

3) Emergency Operations: Ad hoc wireless networks are useful in emergency operations such as search and rescue, and crowd control.

\section{Routing Protocol}

Classification of routing protocol can be done in several ways, but most of the routing protocols are depend on the routing strategy [1]. According to the routing strategy the routing protocols are categorized as: table-driven, source initiated and hybrid.

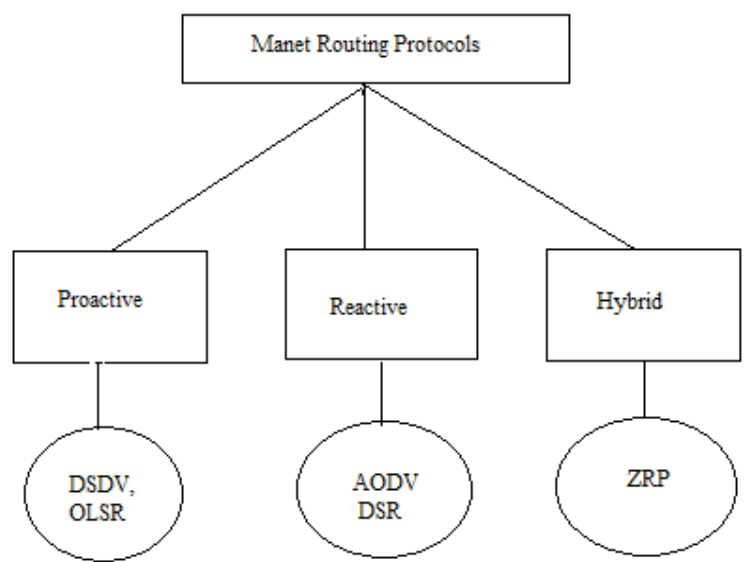

\section{Figure 2. Types of Routing Protocols in MANETs}

\section{A. Table-Driven Routing Protocols(Proactive)}

Table-driven routing protocols are also called as proactive protocols because they maintain the routing information before needed. In the network, each and every node maintains the routing information to every other node. Routing table has routes information and is periodically updated when topology changes in the network. This protocol is not suitable for large network because they need to maintain routing information of each and every node to every other node in the network. This may cause more routing overhead and leads to more bandwidth consumption in the routing table. DSDV, WRP and OLSR come in this category.

\section{B. On Demand Routing Protocols(Reactive)}

These protocols are also called as reactive protocols because they maintain the routing activity on demand or when it is needed at the time of communication. If a node wishes to send a packet to another node then firstly this protocol searches for a route in an on demand manner and established a connection for sending and receiving the packet. The route discovery process occurs by flooding the RREQ packets throughout the network. These protocols maintain the routing information only for active routes. This on demand manner of maintaining routing information causes less routing overhead as compared to table driven routing protocol. DSR, AODV comes under this category. 


\section{Hybrid Routing Protocols}

In this protocol a well combination of both proactive and reactive routing protocols are used including advantages of both protocols. These types of protocols can incorporate the facility of both protocols without compromising its own advantages. ZRP comes under this category.

\section{Related Work}

Finding an energy efficient routing protocol has always been a key challenging issue in mobile ad-hoc networks. Following are the methods to deal with energy conservation to improve networks lifetime.

\section{A. Enhancement of MANET Routing Protocol}

Energy consumption is an important issue as nodes are battery powered and mobile in nature. Hence, it requires energy efficient routing protocols to reduce power consumption and improve the robustness of the system.

This paper [3] evaluates the performance of various ad-hoc routing protocols such as DSDV, AODV, DSR, TORA and AOMDV on the basis of energy efficiency and they also proposed a routing algorithm that not only modifies AOMDV but also give better performance as compared to all the above protocols. Proposed Energy-AOMDV protocol performs better in case of packet delivery ratio (PDR), throughput, jitter and average end to end delay. The packet drop in case of Energy-AOMDV is less than AOMDV.

\section{Conclusion}

This protocol considers residual energy in order to enhance life of network and to minimize energy consumption. We can also consider other metrics i.e. transmission power of nodes, signal strength, stability of the node to find more energy efficient path.

\section{B. Enhanced AODV - An Energy Efficient Routing Protocol for MANET}

This paper [4] presents an EERP-protocol based on AODV. This protocol reduces the transmission power of a node which is a part of an active route if next hop node is closer. A method is proposed the distance between two consecutive nodes is calculated based on RSS (received signal strength) from next hop during route reply process. If RSS is high, it implies nodes are closer; as a result lesser transmission power will be required to transmit data and reduces battery consumption.

\section{Conclusion}

Some parameters are being considered such as residual battery and throughput which is increasing for EERP-protocol. This proposed protocol improves the performance of networks by minimizing the transmission power control.

\section{Energy Efficient Enhanced AODV Routing Protocol for Mobile Ad hoc Network}

In this paper [5] protocol path selection done on the basis of residual energy, traffic density and the stability of the node until the time T. RREP packets are broadcasts along the paths. Power loss level is calculated by subtracting received energy from transmitting energy along the particular path. Data packets are sending through the path having low power loss level.

\section{Improved-AODV Routing Protocol for Mobile Ad hoc Networks}

This paper [6] presents an improved AODV protocol. Stability factor is used to calculate the remaining energy of nodes. The stability of a node is defined as the ratio of its remaining energy to the initial energy [7].

The proposed protocol I-AODV is similar to AODV with some additional constraints on route discovery process. In AODV, remaining energy of node is not considered during 
route discovery process. While in I-AODV, when a source node sends a RREQ message and it reaches to its neighboring node, then that node first checks its stability factor.

In order to reduce end to end delay, I-AODV protocol done three modifications:

- An improved ad hoc routing mechanism is used to find path between source and destination.

- Decreased RREP wait time during the back-off and the predefined TTL threshold value has been modified.

- Expanding ring search has been modified for fast route discovery.

It is observed that by using three techniques in routing protocol it is possible to reduce the energy and end to end delay about $7 \%$ to $24 \%$.

\section{Conclusion}

Energy consumption of network can be reduced by decrementing the transmission power of nodes depending in the minimum distance required for communication and energy level of node. This modification can further improve the existing protocol.

\section{E. Signal Strength Based Route Selection in MANET}

This paper [8] proposed a methodology in which strength of signal is calculated and then compares it with RSSI (receiving signal strength indicator) threshold value. RSSI value is calculated with the help of two ray ground model. If this RSSI value is greater than threshold value then it is accepted else discarded. The advantage of this methodology is that we can increase the network lifetime while selecting a route to destination. The proposed SSAODV have several advantages over AODV in terms of the metrics: end to end delay, routing overhead, packet delivery ratio, and throughput.

\section{Limitation}

This protocol mainly emphasize on signal strength for selecting a route to destination. We can also consider nodes energy with signal strength while selecting a route to destination because nodes have limited battery so it is required to save battery of nodes having low powered to enhance network lifetime. In this way node always choose a route having sufficient energy and signal strength.

\section{F. OAODV Routing Protocol for Improving Energy Efficiency in MANET}

In the traditional AODV, a node floods RREQ message to its neighboring node to find out the path to the destination for communication. If life of intermediate node is less, it could not further broadcast the RREQ packet because node goes down. So, it could not send RREP packet on reverse path. Hence, source node has to rebroadcast RREQ message to find a route to destination. This increases rebroadcasting of RREQ packets, end to end delay and decreases packet delivery ratio.

OAODV based [9] routing protocol modifies the broadcast mechanism of traditional AODV protocol. It maintains a routing table having each node battery life and node density in its surrounding. Two parameters are taken into consideration for broadcasting the RREQ packet i.e. node battery life and node density in its surrounding. In this protocol, a node broadcast the RREQ packet when a node has sufficient battery life and its node density in its surrounding is greater than a particular threshold value. This may decrease unnecessary rebroadcasting of RREQ packets. Hence, proposed OAODV protocol is much better as compared to traditional AODV protocol in terms of battery lifetime and throughput and increases the life of battery.

\section{G. Energy Based AODV Routing Algorithm with Sleep Mode in MANETs}

This paper [10] mainly focuses on increasing the life of node in the network. They proposed a methodology in which, one set the minimum energy threshold limit of a mobile node, if a node energy level reaches the minimum threshold limit then its mode 
changes to sleep mode, saves energy and exists as long as possible in the network. This improves the overall MANETs efficiency.

\section{H. Optimize Signal Strength and Energy Efficient Mechanism for Link Failure in MANET}

This paper [11] proposed a combined technique based on signal strength and energy of node for routing in MANET. The distances between two consecutive nodes are calculated based on RSSI and energy of node is also calculated. If calculated RSSI and energy of node is greater than the predefined threshold value then it is accepted otherwise discards it. This combined technique not only choose route having sufficient energy and signal strength but also improves performance and consumes less energy of nodes in MANET.

\section{Limitation}

This protocol is restricted to only few techniques. By combining more metrics to this protocol we can improve the performance much better and find more optimized path and longer-lived route.

\section{Conclusion}

Mobile Ad hoc network is a collection of mobile nodes and having no central administration. Since, nodes in the network have dynamic topology. As we know that, nodes are battery powered and depend on energy for transmitting or receiving packets in the network. Hence, energy conservation is a key issue in mobile ad hoc network. In this paper, various energy conservation techniques are used, which not only increases the use of energy but also improves the network lifetime. So, the main concern for existence of nodes in the network is to conserve energy. To conserve energy it is necessary to check the remaining energy of node before sending packet through it. In future we will introduce enhancement of energy efficient routing protocol based on AODV in order to conserve more energy.

\section{References}

[1] X. Hong, K. Xu, and M. Gerla, "Scalable Routing Protocols for Mobile Ad hoc Networks", (2002).

[2] K. A Talwar, S. M. Benakappa and B. N. Yuvaraja, "A Survey: Routing Protocols in MANETs", International Journal of Innovative Research in Computer and Communication Engineering, vol. 2, iss. 7, (2014).

[3] R. Khan, A. M. N. Azad. and S. A. Vishwakarma, "Enhancement of MANET Routing Protocol”, 978-14799-3064-7/14/\$31.00, 2014 IEEE.

[4] U. R. Bhatt, P. Jain and R. Upadhyay, "Enhanced AODV - An Energy Efficient Routing Protocol for MANET", 2013Nirma University International Conference on Engineering (NUICONE), IEEE.

[5] R. Rajeshkanna and A. Saradha, "Energy Efficient Enhanced AODV Routing Protocol for mobile Ad hoc Network", IJCTA, vol. 4, (2013).

[6] S. Kurundkar and A. Maidamwar, "Improved-AODV Routing Protocol for Mobile Ad hoc Networks", International Journal of Advanced Research in Electrical, Electronics and Instrumentation Engineering (IJAREEIE), vol. 2, iss. 7, (2013).

[7] S. K. Singh, S. Prakash and K. Kumar, "Energy Aware Dynamic MANET On-demand (EA-DYMO)", International Journal of Computer Applications, (2011).

[8] N. Manjhi and N. Patel, "Signal Strength Based Route Selection in MANETs", International Journal of Computer Science and Telecommunications, vol. 3, iss. 7, (2012).

[9] S. P. Bhatsangave and V. R. Chirchi, "OAODV Routing Protocol for Improving Energy Efficiency in MANET”, International Journal of Computer Applications, vol. 51, no. 21, (2012).

[10] T. Nema, A. Waoo, P. S. Patheja and S. Sharma, "Energy Efficient Adaptive Routing Algorithm in MANET with Sleep Mode", IJACR, vol. 2, no. 4, iss. 6, (2012).

[11] K. Khator and N. Manjhi, "Optimize Signal Strength and Energy Efficient Mechanism for Link Failure in MANET", IJCNWMC, vol. 5, iss. 1, (2015), pp. 1-12. 